\title{
Evaluating a Values-Based Intervention for Adolescence with High Nonclinical Paranoia: A Schools-Based Randomised Control Trial
}

\author{
A. Parker ${ }^{1} \cdot$ J. Kingston ${ }^{1}$ (D) \\ Accepted: 5 November 2021 / Published online: 18 November 2021 \\ (c) The Author(s) 2021
}

\begin{abstract}
Background Paranoia in adolescents is common, but research on attenuating it is scarce. Focusing on values and enhancing value-based acts is a low intensity method for attenuating paranoia in adults. This randomised trial compared a brief (30-min, self-directed) values-plus-goals intervention to an active control for adolescents with high nonclinical paranoia (Paranoia Scale $\geq 53$ ), delivered in schools. The study also investigated the role of self-esteem.

Methods Ninety adolescents were randomly assigned to condition. Paranoia (primary outcome) and self-esteem (potential mediator) were assessed at baseline (T1), and two- (T2) and six-weeks (T3) after baseline.

Results Results were analysed using intention to treat (ITT) and per protocol (PP) analysis. Using ITT analysis, the Condition*Time interaction was significant $\left(F_{(2,168)}=3.98, p=.02\right)$, paranoia was significantly lower at T3 following valuesplus-goals as compared to control $(d=0.64)$. Differences were not significant using per protocol analysis $\left(F_{(2,106)}=1.61\right.$, $p=.21)$. The between group effect size at $\mathrm{T} 3$ was $(d=0.61)$. The Condition*Time interaction for self-esteem was not significant $\left(F_{(2,112)}=2.86, p=.06\right)$.

Conclusions Tentatively, findings suggest that a brief values-plus-goals intervention can reduce paranoia in adolescents relative to an active control.
\end{abstract}

Keywords Values $\cdot$ Goals $\cdot$ Paranoia $\cdot$ Self-esteem $\cdot$ Adolescents

\section{Introduction}

Paranoia describes the unfounded belief that another person is, or is planning to, act in a way to intentionally cause one harm (Freeman \& Garety, 2014). There is now substantial evidence that rare and severe forms of paranoia (i.e., persecutory delusions) exist on a continuum with milder and everyday suspicion and mistrust of others, which is common in the general population (Bebbington et al., 2013; Elahi et al., 2017). Non-clinical paranoia can be distressing, pre-occupying, and associated with feelings of isolation and powerlessness (Ellett et al., 2003; Ellett \& Chadwick, 2007; Freeman et al., 2005, 2011). It can also be a risk factor for developing a psychotic disorder (Poulton et al., 2000). Understanding paranoia in the non-clinical population is therefore important

J. Kingston

Jessica.kingston@rhul.ac.uk

1 Royal Holloway, University of London, Egham, UK in its own right, but may also help to inform the understanding and treatment of persecutory delusions.

Emerging research highlights adolescence as a critical time for experiencing paranoia (Bird et al., 2019), with $20-30 \%$ of adolescents reporting paranoid thoughts on a weekly basis. Put into developmental context this is not surprising, as the specific nature of threat-based cognitions are causally influenced by social context (Weems \& Stickle, 2005). Adolescence is a period of heightened social sensitivity and vulnerability for many teenagers, where concerns about social comparison, failure and criticism are paramount (Warren \& Sroufe, 2004). Experiences of victimisation and bullying are most likely to occur at this time, alongside increased periods of time spent outside of the home with less familiar others. Paranoia in adolescents is distressing in its own right, as well as being a probable antecedent to subsequent persecutory delusions, albeit for a minority (Bird et al., 2019). To date, only a small number of studies have begun to investigate methods for attenuating paranoia in adolescents. This paper reports on the use of a brief, strengths-focused, universal intervention for reducing 
paranoia in adolescents self-reporting high baseline levels, as well as examining possible mechanisms of change.

Many factors have been identified as playing a casual role in the development and persistence of paranoia. The self, and in particular, negative views of the self such as low trait self-esteem (high negative and low positive self-beliefs), unstable state self-esteem (highly fluctuating positive and negative self-beliefs) and negative core beliefs (negative global self-beliefs) have been established as causal mechanisms across the spectrum of experience in adults (e.g., Jongeneel, Pot-Kolder, Counotte, van de Gaag \& Veling, 2018; Kesting et al., 2013) and have been cross-sectionally associated with paranoia in adolescent groups (Bird et al., 2017, 2019; Raes \& Van Gucht, 2009). The way in which negative self-views increase vulnerability for paranoia remains to be fully elucidated and may vary across individuals. One account proposes that negative self-views and/or low positive self-views increase feelings of inferiority and vulnerability of harm from others (Freeman \& Garety, 2014), whilst others argue that paranoid cognitions serve to protect one's self-esteem in the face of self-esteem threats, by attributing the cause of threat to external others (Bentall et al., 2001).

An extensive literature in social psychology demonstrates that clarifying and reflecting on a personal strength or life area that is highly valued, important and meaningful to an individual can help to mitigate the impact of threatening interpersonal experiences. Reflecting on highly valued domains can help to engage a broader and more positive view of the self under threat (Sherman, 2013), increase one's willingness to consider threatening information (Cohen \& Sherman, 2014) and de-couple the threat from meaningful aspects of the self (Wakslak \& Thrope, 2009). It is possible that momentary self-worth becomes less contingent on situational threats and thus more stable over time (Harris et al., 2018). From this perspective, reflecting on a deeply held value may help attenuate paranoia by accessing a broader, values-informed perspective when encountering potentially interpersonally threatening experiences. Investigating values and paranoia, Kingston and Ellett (2014) found that university students who had reflected on a highly valued domain before exposure to a paranoia inducing environment had lower state paranoia following the paranoia inducing environment than an active control group. However, effects were not assessed overtime or outside of the laboratory context and processes of change were not examined.

Reflecting on personal values is also an integral component of Acceptance and Commitment Therapy (ACT, Hayes et al., 1999). Here, in addition to clarifying and reflecting on one's values, individuals are guided in developing and committing to valued-based goals (i.e., chosen acts that are informed by one's values). From an ACT perspective, combining values with commitment to value-based action is integral to durable benefits. Informed by this, Evans et al.
(2019) compared the effects of a brief values clarification and reflection intervention on paranoia, with and without the addition of value-based goal setting (i.e., values only versus values-plus-goals). Results indicated that values-plus-goals led to significantly greater reductions in paranoia over a twoweek period. Moreover, moderation analyses identified that the values-plus-goals intervention was especially effective for those with low baseline self-esteem, perhaps suggesting that values-plus-goals "offset the vulnerability towards state paranoia that is otherwise characteristic in individuals with low self-esteem" (p. 5). Extending this work into participants with high nonclinical paranoia, Davies et al. (2021) reported that values-plus-goals was superior to goals-only in reducing high nonclinical paranoia. Davies et al. also investigated whether positive/negative self-views following a difficult interpersonal event were higher/lower following values-plus-goals relative to goals-only. Mean level of positive self-evaluations were higher following the values-plusgoals condition, but differences were not significant, perhaps due to the small sample size.

Overall, therefore, existing research has suggested that clarifying and reflecting on a meaningful value and setting value-based goals can reduce paranoia in individuals with nonclinical (Kingston \& Ellett, 2014; Evans et al., 2019) and high nonclinical (Davies et al., 2021) baseline levels. The primary aim of this study was to test whether these outcomes can be replicated in adolescents, when delivered as a universal intervention in the classroom setting, and when assessed over a 6-week period. This was tested using a randomised control trial, comparing values-plus-goals to an active control, which mimicked some aspects of values-plus-goals (reading about and ranking values, writing a short essay), but crucially involved writing about something that the individual did not value and from the perspective of someone else. Theoretically, from both perspectives, values-plus-goals may attenuate paranoia by helping people with a vulnerability to paranoia to access a broader, values-informed perspective when encountering a potentially threatening interpersonal experience. This may help individuals to maintain a positive view of the self in situations that may otherwise reduce selfesteem and increase feelings of inferiority and vulnerability of harm from others. With this in mind, the secondary aim of the current study was to test whether the values-plus-goals (henceforth called $\mathrm{V}+\mathrm{G}$ ) intervention improved adolescents self-reported self-esteem and, if so, whether this mediated any between group effects on paranoia. It was hypothesised that self-esteem would be enhanced in the $\mathrm{V}+\mathrm{G}$ group relative to controls and that this would mediate the effect of condition on $\mathrm{T} 3$ paranoia. 


\section{Methods}

\section{Design}

An experimental design was used, randomising adolescents to one of two conditions: a brief values-plus-goals condition or an active control (controlling for the values ranking task, brief essay writing, and repeated measurement). The dependent variable was paranoia and the mediating variable was self-esteem. Each were measured at baseline (T1), at the end of the intervention phase (two weeks after baseline, T2), and six-weeks after baseline assessment (T3).

\section{Participants \& Procedures}

Two hundred and ninety-six adolescents (14-16 years old) were recruited from secondary schools in the inner and outer London area. Initial email or telephone contact was made with all local authority mainstream schools and academies with pupils aged 14-16 across 17 London boroughs. Further schools were contacted opportunistically in Hertfordshire, Surrey and Oxfordshire. Four schools agreed to participate (school 1 and 4 were inner-city). All participants were invited to take part in the study; however, only the data of those who exceeded a pre-established cut-off score of 1SD above the nonclinical norm on the Paranoia Scale (Combs \& Penn, 2004; Fenigstein \& Vanable, 1992) are reported on. Ninety participants had baseline paranoia score that exceeded the cut-off. An a priori power analysis using $\mathrm{G}^{*}$ POWER indicated that a total sample of $n=54$ ( 27 per condition) was required to provide $95 \%$ statistical power with $\alpha=.05$ and detecting a medium-large $(f=.30)$ effect size of condition allocation on paranoia, based on previous research (e.g., Davies et al., 2021; Evans et al., 2019). Twenty-eight percent of the high nonclinical paranoia sample were male and the mean age was 14.5 years $(S D=.52)$. Fifty-four percent identified as White British, 19\% as Black, 12\% mixed race, $11 \%$ Asian and $3 \%$ other.

The study was reviewed and approved by the host university's Ethical Committee before the study commenced. Questionnaire packs and tasks were randomised by condition and questionnaire order in advance by a second researcher not otherwise involved in the research and placed in sealed envelopes labelled with a letter string unrelated to the participant ID number inside the pack. These packs were distributed to participants pre-sealed with the researcher aware only of the external letter string and the participant name, but unable to match this to the participant ID number or condition enclosed. Packs were randomised in batches for each school in an attempt to match numbers across conditions. Consenting participants completed demographic and baseline measures before completing the $\mathrm{V}+\mathrm{G}$ or control task. The initial tasks took approximately 30 -min to complete and were fully self-directed. Two-weeks later, participants completed a second set of questionnaires and indicated whether they had completed their goal (V+G only). Six-weeks later, participants completed a final set of questionnaires and were thanked and debriefed. All measured and tasks were completed in classroom settings.

\section{Measures}

All measures and intervention tasks were provided to participants in paper format within group settings (i.e., in the classroom). The order of presentation of self-report questionnaires was randomised at each time point to minimise potential order effects.

\section{Primary Outcome Measure}

Paranoia Scale (Fenigstein \& Vanable, 1992) is a 20-item questionnaire designed to measure non-clinical paranoid thoughts (e.g., "Someone has it in for me"; "I sometimes feel as if I am being followed"). Items are rated from $l=n o t$ at all applicable to me to $5=$ extremely applicable to me and total scores range from 20 to 100, with higher scores indicating higher levels of paranoia. The PS is a well-recognised method for detecting subclinical paranoid thoughts (Freeman et al., 2008), with good internal reliability in adolescent groups ( $\mathrm{a}=.89$; Raes \& Van Gucht, 2009), construct validity, and test-retest reliability $(\mathrm{a}=.70)$ over 6 -months period (Fenigstein \& Vanable, 1992). The PS has also been found to be sensitive to change in a non-clinical adolescent group (Campbell \& Morrison, 2007). In this sample, Cronbach's alpha was acceptable at T1 $(\alpha=.65)$ and good at T2 and T3 ( $\alpha=.90$ and .86 respectively).

\section{Potential Mediator}

Self-esteem was measured using the Rosenberg Self-Esteem Scale (Rosenberg, 1965), a 10-item measure ("I feel that I have a number of good qualities" "I take a positive attitude toward myself") rated on a 4-point Likert-scale from $0=$ strongly agree to $3=$ strongly disagree, with total scores ranging from 0 to 30 . The measure has been widely used, including in adolescent populations (e.g. Rosenberg et al., 1989) and reports good psychometric properties. In adult populations, the measure has been shown to have good internal consistency $(\alpha=.92)$ and test-restest reliability of $r=.85$ (Rosenberg, 1979). In the present study, baseline self-esteem demonstrated acceptable internal consistency $(\alpha=.78)$. 
Norms published for adolescents aged 15-17 (Gabhainn \& Mullan, 2003) give a mean score of $27.3(\mathrm{SD}=3.5)$.

\section{Conditions}

Participants in $\mathrm{V}+\mathrm{G}$ began by reading a brief description of values (e.g. Chase et al., 2013). Several descriptions were given, such as "what matter to you in the big picture, what you want to stand for, and the personal qualities you want to develop". Participants were then given 11 value domains (e.g., relations with friends/family, creativity, spontaneity/living life in the moment; see Sherman et al., 2000) to rank ordered according to personal importance. After this, they wrote for ten minutes about the most important value explaining why it was important and meaningful to them, and giving an example of an occasion when it made them feel good about themselves. Subsequently, participants listed two reasons why this value was most important to them and indicated agreement with four statements designed to measure the amount of importance and influence of this value on their life (e.g., "This value or personal characteristic has influenced my life" using a 6-point scale of 1-strongly disagree to 6-strongly agree; Evans et al., 2019). Next, participants were guided in setting a value-based goal to complete in the following two weeks. Participants recorded the goal on their response sheet and took a copy home as a reminder. Two weeks later, participants in this condition were asked to indicate if they had completed the previously set goal.

Participants in the control group received the same information about values and completed the same ranking exercise. However, after ranking their values, control participants were asked to complete a ten-minute writing task in which they described why their least important value may be meaningful to a typical student at their school (see Evans et al., 2019). Participants were encouraged to highlight a particular occasion when this value may have made the typical student feel good about themselves. Subsequently, the participant was asked to list the top two reasons this value might have been chosen by the typical student as their most important value, and to indicate agreement with four statements reflecting on the importance and influence of this value on the typical student. The control condition featured no goal setting task.

\section{Analysis Strategy}

Firstly, to assess the success of randomisation, between group comparisons were computed for sociodemographic and study variables at baseline using independent $t$-tests and Pearson $\chi^{2}$-tests. Similarly, dropout, defined as those participants not completing either of the post-treatment assessments, were compared to those completing it, to assess systematic attrition. We also tested whether baseline paranoia and/or self-esteem differed as a function of school and included school as a covariate (using dummy coding) where relevant.

Secondly, the primary hypothesis was tested using a 2 (condition: $\mathrm{V}+\mathrm{G}$ vs control) * 3 (time: $\mathrm{T} 1, \mathrm{~T} 2$ and $\mathrm{T} 3$ ) repeated measures ANCOVA (controlling for school and age, see results). Next, post-hoc ANCOVAs were computed to examine between-group differences at T2 and T3, controlling for T1. To assess the size of the between group effect in a manner comparable to other intervention studies, cohen's $d$ was computed.

Due to drop-out overtime (see Fig. 1), the primary hypothesis was tested two ways. Firstly, intention to treat (ITT) analysis was computed. Here, all participants were included in the analyses regardless of whether they dropped out at T2/T3 (i.e., $n=90$ ). The scores of those who had dropped out of the study were estimated using Multiple Imputation (MI) and Predictive Mean Matching in SPSS, with 10 iterations (computed per condition) calculated and averaged. Multiple imputations were conducted on each condition separately in order to limit the impact on any potential interactions between groups (Sullivan et al., 2018). Secondly, we analysed the data using per protocol analyses. Here, the analyses were the same as those described for ITT, but only those participants who completed all phases of the study were included in the analyses (i.e., $n=35 / 36 \mathrm{~V}+\mathrm{G}$ and $n=24 / 23^{1}$ control). The aim of the per protocol analysis was to examine the effects of the conditions when administered to the target sample.

Finally, to examine the effects of condition on selfesteem, a 2 (condition: $\mathrm{V}+\mathrm{G}$ vs control) * 3 (time: $\mathrm{T} 1, \mathrm{~T} 2$ and T3) repeated measures ANCOVA was computed, with post-hoc ANCOVAs at T2 and T3 (controlling for T1) to probe a significant interaction. Mediation analyses were planned if the interaction was significant, using PROCESS model 4 and entering T2 self-esteem as the mediator, T3 paranoia as the outcome variable, condition as the independent variables, and controlling for age and baseline self-esteem and paranoia.

\section{Results}

\section{Descriptives and Preliminary Analyses}

The flow of participants through the study is depicted in Fig. 1. Means and standard deviations are presented in Table 1. Ninety participants completed baseline

\footnotetext{
${ }^{1}$ Numbers differ slightly for paranoia scale (first number) and selfesteem (second number).
} 
Fig. 1 CONSORT diagram outlining participant flow through the study. $V+G$ valuesplus-goals, $P S$ paranoia scale, $I T T$ intention to treat, $P P$ per protocol. ${ }^{1}$ Invited to take part in study, but screened out of analyses doe scoring $<53$ on Paranoia Scale. *Numbers differ for paranoia scale/self-esteem scale.** PP 'n' based on participants completing all time points

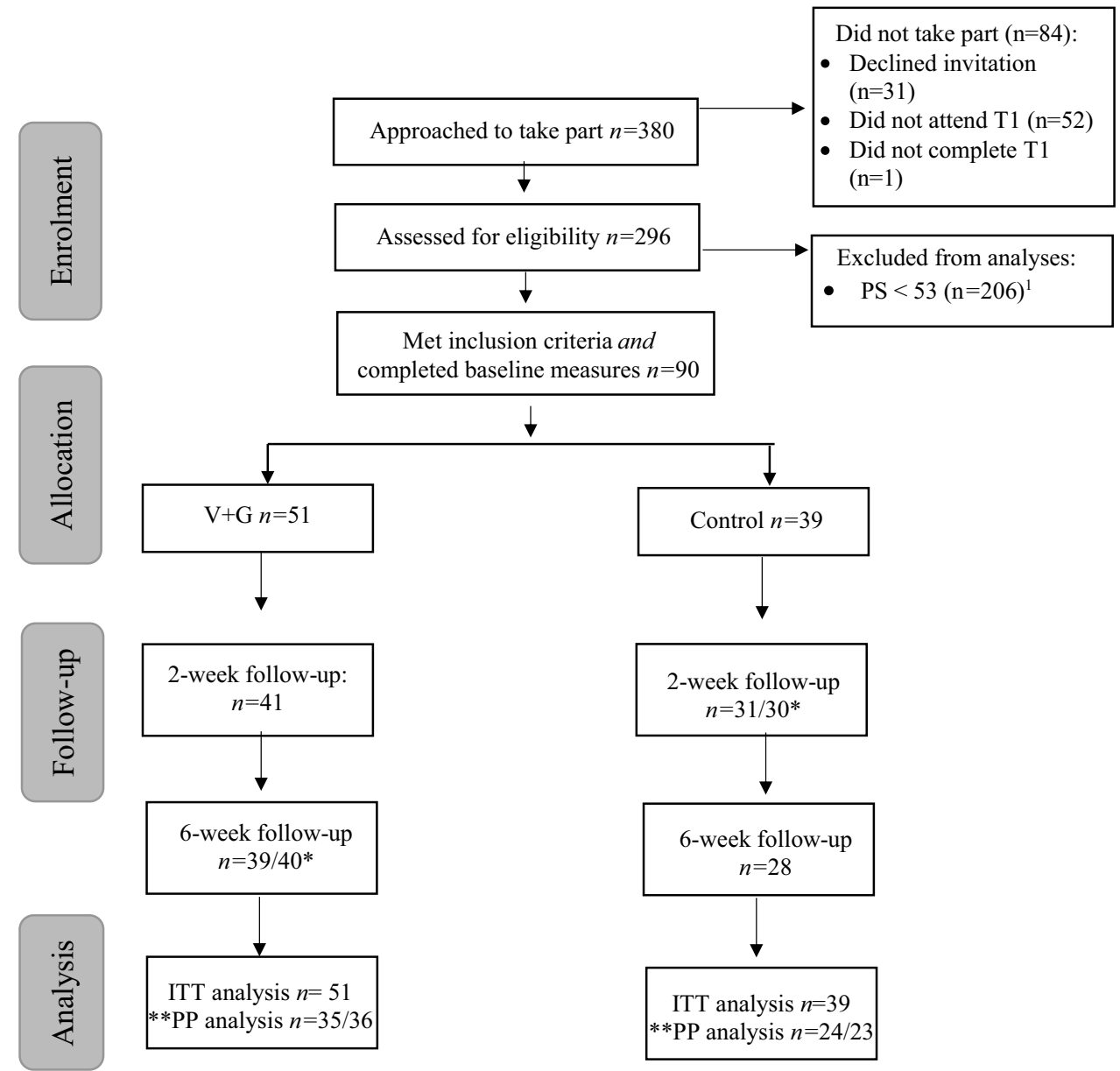

assessment and met inclusion criteria. Paranoia scores ranged from 53 to $100, \mathrm{M}=62.97(\mathrm{SD}=8.90)$. Due to skew and outliers, paranoia scores were square-root transformed; however, outcomes from hypothesis testing were unchanged when using transformed and untransformed data. Untransformed are therefore presented. For instances where $<10 \%$ of data was missing (per participant) for a given scale, the score was estimated using the mean score for that participant on the questionnaire at that time point.

Comparing $\mathrm{V}+\mathrm{G}$ versus control on baseline characteristics indicated that participants in the control group were older than those in the $\mathrm{V}+\mathrm{G}$ condition $(t(88)=-2.59$, $p=.011)$. Age was therefore co-varied in the analyses. The distributions of participants from across the four participating schools were: school $1(n=26)$, school $2(n=46)$, school $3(n=13)$ and school $4(n=5)$. Comparing baseline paranoia and self-esteem scores across schools indicated a suggestive trend towards higher paranoia in school 1 relative to 2 and 4 ( $p$ s $<.08)$. School was dummy coded and controlled for in paranoia analyses, using school 2 (highest n) as the reference school. Comparing those who completed versus dropped-out of the study indicated that there were no systematic differences across baseline variables or socio-demographic variables $(t \mathrm{~s}<1.15$, ps $>.05 ; \chi<5.38$, ps $>.05)$.

Forty-six percent of participants in the $\mathrm{V}+\mathrm{G}$ condition reported completing their value-based goal when asked at 2-week follow-up. An ANCOVA comparing T3 paranoia scores for those completing their valued-goals versus those not completing them, whilst controlling for baseline paranoia, showed no significant differences (completed goal, $\mathrm{M}_{\text {para }}=55.5$; goal not completed $\mathrm{M}_{\text {para }}=54.10 ; t(25)=.452$, $p=.655)$. Consistent with existing research, paranoia was negatively associated with self-esteem at baseline $(r=-.227, p=.031)$.

\section{Main analysis: Paranoia}

\section{Intention-to-Treat Analysis}

The Condition*Time interaction was significant $\left(F_{(2,168)}=3.98, p=.02, \eta \mathrm{p}^{2}=.045\right)$, suggesting a differential change in paranoia scores as an effect of condition. There was also a significant school $1 *$ Time interaction, suggesting that paranoia scores in school 1 differed from the reference group, overtime $\left(F_{(1,83)}=6.31, p=.002, \eta p^{2}=.07\right)$. 
Interactions were decomposed using ANCOVAs, comparing paranoia scores at T2 and at T3, whilst controlling for baseline paranoia, school 1 and age. Conditions did not differ on paranoia scores at $\mathrm{T} 2\left(F_{(1,85)}=.169, p=.682\right)$, but paranoia was significantly lower in the $\mathrm{V}+\mathrm{G}$ group as compared to control at T3 $\left(\mathrm{F}_{(1,85)}=4.65, p=.034\right)$. Controlling for baseline differences in paranoia, the between group effect size at T2 was $d=0.19$ and T3 $d=0.64$. Baseline paranoia also significantly predicted paranoia at T2 $\left(F_{(1,83)}=20.77, p<.001\right)$ and T3 $\left(F_{(1,83)}=12.96, p=.001\right)$.

\section{Per Protocol Analysis}

Conducting the same analyses in those participants completing all three time points indicated no Condition*Time interaction $\left(F_{(2,106)}=1.61, p=.205\right)$. Controlling for baseline differences in paranoia, the between group effect size at T2 was $d=0.02$ and at T3 $d=0.61$.

\section{Mediation Analysis}

A repeated measures ANCOVA was computed to test for between group differences in self-esteem, overtime, whilst controlling for age. The Time*Condition interaction was suggestive, but did not reach significance $\left(F_{(2,112)}=2.86\right.$, $p=.062$ ).

\section{Discussion}

The current study examined the relative effects of a brief values-plus-goals intervention versus an active control on paranoia scores in adolescents with high nonclinical paranoia, assessing paranoia two and six weeks post task. Using intention to treat and per protocol analyses indicated no between group differences at two-weeks, but a medium level difference at six-weeks (i.e., $d=.64$ and $d=.61$ respectively), in favour of the values-plus-goals intervention. This was statistically significant in the intention to treat analysis but not in the per protocol analysis, perhaps due to the smaller sample size in the latter. Values-plus-goals and the control were matched with regards to reading a short introductory paragraph about values, ranking one's values from most to least important, writing a brief essay, and completing self-report measures over three time points. Conditions differed in two specific ways: the values-plus-goals condition included focused reflection on an autobiographical memory that exemplified that value and involved guidance on and actively setting a goal to help them live in accordance with that value. Conversely, control participants wrote about something they least valued and from the perspective of another and did not think about or set goals. This could suggest that the elements responsible for the differential 
effects were personal reflection on a highly valued domain (rather than simply value ranking) and thinking about and setting goals in accordance with this value. This replicates previous research in a predominantly university student samples, which identified that the combining of reflection and goal setting was superior to either in isolation (Davies et al., 2021; Evans et al., 2019).

These findings are important for a number of reasons. The findings suggest that the effectiveness of a $V+G$ intervention for reducing high nonclinical paranoia is replicable in an adolescent sample (Davies et al., 2021). Adolescence has been described as a "window of opportunity" for intervening and offsetting negative developmental trajectories (Cicchetti \& Rogosch, 2002), and yet is also characterised as a developmental period where engaging individuals in psychological intervention can be especially challenging (Sauter et al., 2009). The universal approach, afforded by strengths-based interventions, is especially well suited to helping young people in a destigmatising way and could easily be replicated by schools. It is also important to note that these effects were replicated in a sample who were more socio-demographically diverse than previous, predominantly university student, samples (i.e., in terms of educational ability, ethnicity, and race) with results demonstrating a medium controlled effect size over a 6-week period.

Contrary to expectation, we did not find a significant interaction for self-esteem. Whilst means suggested an increase in self-esteem that was specific to the $V+G$ group, the interaction fell short of statistical significance (i.e., $p=.062$ ), perhaps due to the small sample size. Future research would benefit from examining the possible processes underpinning the effects of values-plus-goals on paranoia using samples powered for mediation analyses. In doing so, it could be useful to explore process variables that underpin values work in Acceptance and Commitment Therapy, such as improved psychological flexibility (i.e., an ability to engage in and pursue valued behaviour even when experiencing emotional discomfort, see Cherry et al., 2021). Bird et al. (2017) have also shown that affect is a probable driver of paranoia in adolescents and it is possible that thinking about core values and engaging in valued acts could help to improve paranoia via reduced negative affect.

The current findings should be considered in the light of some limitations. Findings were based purely on self-report which rely on the participant's ability to accurately capture their experiences. Future research would benefit from using real time behavioural assessments of the impact of values on paranoia, such as through the use of virtual reality, experience sampling methods, or through the observation of participant's behaviour in social scenarios. Relatedly, this study only examined the frequency of experiencing paranoid cognitions. Paranoia is a multifaceted experience and future research would benefit from examining belief dimensions such as distress, conviction and impact on functioning. Also related to this, Cronbach's alpha was low (.65), albeit acceptable, for T1 paranoia scores. Interestingly, values were good at $\mathrm{T} 2$ and $\mathrm{T} 3$, but nonetheless this suggests suboptimal interrelatedness between items at this particular point in time. Finally, whilst the follow-up period of 6-week exceeds that of previous research, the durability of effects over a longer period of time is an important direction for future research.

Notwithstanding these limitations, the study has some tentative implications. In conjunction with previous work (Davies et al., 2021), the findings suggest that a brief values clarification, reflection and values-based goal setting intervention can be effectively delivered in schools, in a low intensity and universal way and that it can improve feelings of mistrust and suspiciousness towards others in adolescents. The method of delivery was inexpensive and could be integrated into personal support curriculums without the need for advanced staff training. Furthermore, the universal mode of delivery meant that children did not need to be identified as having difficulties in order to benefit and, in this way, the values intervention offered a de-stigmatising method of intervention. The findings also suggest that the effects of this brief intervention were durable over a six-week follow-up period.

Funding This study was not funded.

\section{Declarations}

Conflict of Interest A. Parker and J. Kingston have No conflicts to declare.

Informed Consent and Animal Rights Informed consent was obtained from all individual participants included in the study. No animals were involved in the study.

Open Access This article is licensed under a Creative Commons Attribution 4.0 International License, which permits use, sharing, adaptation, distribution and reproduction in any medium or format, as long as you give appropriate credit to the original author(s) and the source, provide a link to the Creative Commons licence, and indicate if changes were made. The images or other third party material in this article are included in the article's Creative Commons licence, unless indicated otherwise in a credit line to the material. If material is not included in the article's Creative Commons licence and your intended use is not permitted by statutory regulation or exceeds the permitted use, you will need to obtain permission directly from the copyright holder. To view a copy of this licence, visit http://creativecommons.org/licenses/by/4.0/.

\section{References}

Bebbington, P. E., McBride, O., Steel, C., Kuipers, E., Radovanoviĉ, M., Brugha, T., .. \& Freeman, D. (2013). The structure of 
paranoia in the general population. The British Journal of Psychiatry, 202, 419-427

Bentall, R. P., Corcoran, R., Howard, R., Blackwood, N., \& Kinderman, P. (2001). Persecutory delusions: A review and theoretical integration. Clinical Psychology Review, 21(8), 1143-1192. https://doi. org/10.1016/S0272-7358(01)00106-4

Bird, J. C., Waite, F., Rowsell, E., Fergusson, E. C., \& Freeman, D. (2017). Cognitive, affective, and social factors maintaining paranoia in adolescents with mental health problems: A longitudinal study. Psychiatry Research, 257, 34-39. https://doi.org/10.1016/j. psychres.2017.07.023

Bird, J. C., Evans, R., Waite, F., Loe, B. S., \& Freeman, D. (2019). Adolescent paranoia: Prevalence, structure, and causal mechanisms. Schizophrenia Bulletin, 45(5), 1134-1142. https://doi.org/ $10.1093 / \mathrm{schbul} / \mathrm{sby} 180$

Campbell, M. L. C., \& Morrison, A. P. (2007). The relationship between bullying, psychotic-like experiences and appraisals in 14-16-year olds. Behaviour Research and Therapy. https://doi. org/10.1016/j.brat.2006.11.009

Chase, J. A., Houmanfar, R., Hayes, S. C., Ward, T. A., Vilardaga, J. P., \& Follette, V. (2013). Values are not just goals: Online ACT-based values training adds to goal setting in improving undergraduate college student performance. Journal of Contextual Behavioral Science, 2(3-4), 79-84. https://doi.org/10.1016/j.jcbs.2013.08.002

Cherry, K., Hoeven, E. V., Patterson, T. S., \& Lumley, M. N. (2021). Defining and measuring "psychological flexibility": A narrative scoping review of diverse flexibility and rigidity constructs and perspectives. Clinical Psychology Review, 84, 101973. https://doi. org/10.1016/j.cpr.2021.101973

Cicchetti, D., \& Rogosch, F. (2002). A developmental psychopathology perspective on adolescence. Journal of Consulting and Clinical Psychology, 70, 6-20.

Cohen, G. L., \& Sherman, D. K. (2014). The Psychology of Change: Self-Affirmation and Social Psychological Intervention. Annual Review of Psychology, 65(1), 333-371. https://doi.org/10.1146/ annurev-psych-010213-115137

Combs, D. R., \& Penn, D. L. (2004). The role of subclinical paranoia on social perception and behavior. Schizophrenia Research, 69(1), 93-104. https://doi.org/10.1016/S0920-9964(03)00051-3

Davies, M., Ellett, L., \& Kingston, J. (2021). A randomised comparison of values and goals, versus goals only and control, for high nonclinical paranoia. Cognitive Therapy and Research, 45, 1213-1221.

Elahi, A., Algorta, G. P., Varese, F., McIntyre, J. C., \& Bentall, R. P. (2017). Do paranoid delusions exist on a continuum with subclinical paranoia? A multi-method taxometric study. Schizophrenia Research, 190, 77-81. https://doi.org/10.1016/j.schres.2017.03. 022

Ellett, L., \& Chadwick, P. (2007). Paranoid cognitions, failure, and focus of attention in college students. Cognition and Emotion, $21,558-576$.

Ellett, L., Lopes, B., \& Chadwick, P. (2003). Paranoia in a nonclinical population of college students. The Journal of Nervous and Mental Disease, 191, 425-430.

Evans, N., Ellett, L., Carpenter, R., \& Kingston, J. (2019). Immediate and short term effects of values-based interventions on paranoia. Journal of Behavior Therapy and Experimental Psychiatry, 65, 101500. https://doi.org/10.1016/j.jbtep.2019.101500

Fenigstein, A., \& Vanable, P. A. (1992). Paranoia and self-consciousness. Journal of Personality and Social Psychology, 62(1), 129_ 138. https://doi.org/10.1037/0022-3514.62.1.129

Freeman, D., Garety, P. A., Bebbington, P. E., Smith, B., Rollinson, R., Fowler, D., \& Kuipers, E. (2005). Psychological investigation of the structure of paranoia in a non-clinical population. British Journal of Psychiatry, 186, 427-435. https://doi.org/10.1192/bjp. 186.5.427.
Freeman, D., Bentall, R., \& Garety, P. (2008). Persecutory Delusions: Assessment, Theory and Treatment. Oxford University Press.

Freeman, D., McManus, S., Brugha, T., Meltzer, H., Jenkins, R., \& Bebbington, P. (2011). Concomitants of paranoia in the general population. Psychological Medicine, 41(5), 923-936. https://doi. org/10.1017/S0033291710001546.

Freeman, D., \& Garety, P. (2014). Advances in understanding and treating persecutory delusions. Social Psychiatry and Psychiatric Epidemiology, 49, 1179-1189.

Gabhainn, S., \& Mullan, E. (2003). Self-esteem norms for Irish young people. Psychological Reports, 92(3), 829-830. https://doi.org/ 10.2466/pr0.2003.92.3.829

Harris, P., Griffin, D., Napper, L., Bond, R., Schuz, B., Stride, C., et al. (2018). Individual differences in self-affirmation: Distinguishing self-affirmation from positive self-regard. Self and Identity, 18(6), 589-630. https://doi.org/10.1080/15298868.2018.1504819.

Jongeneel, A., Pot-Kolder, R., Counotte, J., van der Gaag, M., \& Veling, W. (2018). Self-esteem moderates affective and psychotic responses to social stress in psychosis: A virtual reality study. Schizophrenia Research, 202, 80-85. https://doi.org/10.1016/j. schres.2018.06.042

Kesting, M. L., Bredenpohl, M., Klenke, J., Westermann, S., \& Lincoln, T. M. (2013). The impact of social stress on self-esteem and paranoid ideation. Journal of Behavior Therapy and Experimental Psychiatry, 44(1), 122-128. https://doi.org/10.1016/j.jbtep.2012. 07.010

Poulton, R., Caspi, A., Moffitt, T. E., Cannon, M., Murray, R., \& Harrington, H. (2000). Children's self-reported psychotic symptoms and adult schizophreniform disorder: A 15-year longitudinal study. Archives of General Psychiatry, 57, 1053-1058.

Raes, F., \& Van Gucht, D. (2009). Paranoia and instability of selfesteem in adolescents. Personality and Individual Differences, 47(8), 928-932. https://doi.org/10.1016/j.paid.2009.07.017

Rosenberg, M. (1965). Society and the Adolescent Self-Image. Princeton University Press.

Rosenberg, M. (1979). Conceiving the self. Basic Books.

Rosenberg, M., Schooler, C., \& Schoenbach, C. (1989). Self-esteem and adolescent problems: modeling reciprocal effects. American Sociological Review. https://doi.org/10.2307/2095720

Sauter, F., Heyne, H., \& Westenberg, M. (2009). Cognitive behavior therapy for anxious adolescents: Developmental influences on treatment design and delivery. Clinical Child and Family Psychological Review, 12, 310-335.

Sherman, D. K. (2013). Self-affirmation: Understanding the effects. Social and Personality Psychology Compass, 7(11), 834-845. https://doi.org/10.1111/spc3.12072.

Sherman, D. K., Nelson, L. D., \& Steele, C. M. (2000). Do messages about health risks threaten the self? increasing the acceptance of threatening health messages via self-affirmation. Personality and Social Psychology Bulletin, 26, 1046-1058. https://doi.org/ 10.1177/01461672002611003

Sullivan, T. R., White, I. R., Salter, A. B., Ryan, P., \& Lee, K. J. (2018). Should multiple imputation be the method of choice for handling missing data in randomized trials? Statistical Methods in Medical Research, 27(9), 2610-2626. https://doi.org/10.1177/09622 80216683570

Wakslak, C., \& Thrope, Y. (2009). Cognitive consequences of affirming the self: The relationship between self-affirmation and object construal. Journal of Experimental Social Psychology, 45, 927-932.

Warren, S. L., \& Sroufe, L. A. (2004). Developmental issues. In T. H. Ollendick \& J. S. March (Eds.), Phobic and anxiety disorders in children and adolescents: A clinician's guide to effective psychosocial and pharmacological interventions (pp. 92-115). Oxford University Press.

Weems, C. F., \& Stickle, T. R. (2005). Anxiety disorders in childhood: Casting a nomological net. Clinical Child and 
Family Psychology Review, 8(2), 107-134. https://doi.org/10. 1007/s10567-005-4751-2
Publisher's Note Springer Nature remains neutral with regard to jurisdictional claims in published maps and institutional affiliations. 